\title{
Virtobot - A Robot System for Optical 3D Scanning in Forensic Medicine
}

\author{
Robert BREITBECK ${ }^{\star a}$, Wolfgang PTACEK ${ }^{b}$, Lars EBERT ${ }^{\mathrm{a}}$, \\ Martin FÜRST ${ }^{b}$, Gernot KRONREIF ${ }^{b}$, Michael THALI

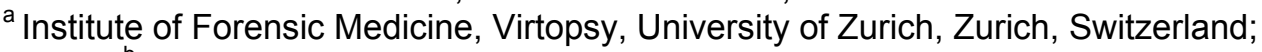 \\ ${ }^{\mathrm{b}}$ ACMIT - Austrian Center for Medical Innovation and Technology, \\ Integrated Microsystems Austria $\mathrm{GmbH}$, Wiener Neustadt, Austria \\ http://dx.doi.org/10.15221/13.084
}

\begin{abstract}
In this article, we present the second prototype of a robotic system, for use in forensic medicine. Among others, the system is able to perform automated whole body surface and wound documentations by using digital close-range photogrammetry and optical 3D surface scanning. Based on the 3D data collected, analysis and reconstruction of forensically relevant events, such as traffic accidents, criminal assaults or homicides can be performed. The newly applied high-tech methods can provide new knowledge about a case, compared to traditional methods.

The purpose of the Virtobot project is to develop a robot prototype that provides fast and accurate, 3D measurement capabilities for documenting external injuries of corpses in three-dimensions and color, supporting forensic investigations.

The system is routinely used at our institute for surface documentation, resulting in 24 surface documentations over the course of 11 months. The main focus was on streamlining the workflow and increasing the level of automation, reducing the scanning times from approximately 40 to 11 minutes. This makes the Virtobot a potentially valuable tool for case documentation in the field of virtual

Reywopsy. virtopsy, forensic imaging, surface scanning, photogrammetry, post mortem, biopsy, tissue sampling, liquid sampling, CT, robotic, radiology, autopsy
\end{abstract}

\section{Introduction}

Besides the post-mortem computed tomography (CT) and magnetic resonance imaging (MRI) for the documentation and diagnostics of internal findings, photogrammetry in combination with 3D surface scanning is used to report external body findings. According to Baglivo et al, only $3 \%$ of all scientific articles in the field of forensic imaging are on post-mortem surface documentation. According to the authors, 3D surface scanning is not yet fully appreciated in forensic investigations although the application of these 3D documentation techniques enables gathering of new data in forensic medical cases [1]. Subsequent virtual 3D reconstructions can help to determine the course of events during an accident or a criminal incident, based on real data [2-10].

While it is possible to generate 3D models from the body out of CT data, the resolution is limited by the slice thickness $(0.6 \mathrm{~mm}$ at best) and color information will not be provided. Therefore highly precise 3D surface scanning, coupled with digital close-range photogrammetry is the gold standard in documenting superficial wounds for reconstructive purposes [11, 12]. Up to now, a series of photographs and surface scans from a variety of perspectives have to be made manually by two experienced operators to perform data collection. This is a time consuming and repetitive task and therefore an ideal task for a robotic system.

In 2003, the vision of an automated system for post-mortem examinations was presented for the first time to the National Institute of Health in Washington DC/Bethesda. This system was envisioned to have imaging components (CT, MRI, MicroCT/MR, 3D surface scanner) as well as a sampling apparatus for histological examinations. In order to perform a broad range of repetitive tasks with both, high speed and accuracy, in the field of forensic medicine, the first multifunctional robotic system named Virtobot was introduced at the Institute of Forensic Medicine in Bern, Switzerland in 2007 [13]. This system was capable of performing fully automated 3D surface scans using an optical surface scanner and CT-guided placement of co-axial introducer needles for tissue or liquid sampling.

This paper describes the current status of the surface documentation feature of second version of the Virtobot, installed 2012 in Zurich.

\footnotetext{
*robert.breitbeck@virtopsy.com; www.virtopsy.com
} 


\section{Materials and Methods}

\subsection{Hardware}



Fig 1. Virtobot room. a: X-Ray shielded working area; b: CT-gantry; c: CT table; d: external rail of the Virtobot; e: Virtobot mounted on external axis with lifting mechanism; $f$ : tool stand with surface scanner, digital photo camera and biopsy module; $g$ : monitor which is connected to the computer running the Virtobot software; $h$ : safety light fence for device protection.

The Virtobot system was developed by Integrated Microsystems Austria GmbH (Wiener Neustadt, Austria). A six-axis robotic arm (Stäubli TX90L, Stäubli International AG, Pfäffikon/CH) is combined with a $4 \mathrm{~m}$ long ceiling-mounted linear guide unit, which is located above the existing CT-gantry, and aligned with the CT table (Figure 1).The system had to be adapted to the given circumstances such as the given room dimensions $6.5 \times 4.1 \times 3.6 \mathrm{~m}(\mathrm{LxWxH})$ with suspended ceiling and the fixed position of the CT system. Therefore the base of the robotic arm is mounted onto a dedicated pneumatic lifting axis (lifting stroke $200 \mathrm{~mm}$ ) to allow moving the robotic arm to a parking position above the CT-gantry in order to keep the general working area free of system components. The input/output terminal box, the main safety controllers and the robot controller box are mounted above the suspended ceiling. The main cabinet, including the system power supply and the controller for the linear axis, is placed in a neighboring engineering room. Behind the X-Ray shielded working area the control station computer is located, which controls the complete system workflow and provides user interaction. A fully automated quick-change system (Schunk SWS40, SCHUNK GmbH \& Co. KG, Germany) allows the robotic manipulator to automatically pick up corresponding end-effectors from the tool magazine station to perform different tasks of the Virtopsy workflow. We currently use three interchangeable end-effector modules: a precise optical 3D surface scanner (GOM ATOS Compact Scan 5M, Gesellschaft für optische Meßtechnik GOM GmbH, Braunschweig/D), a digital SLR camera (Nikon D700, Nikon Corporation, Tokyo/JP) for photogrammetry, as well as a custom-made needle placement tool (Integrated Microsystems Austria GmbH, Wiener Neustadt/A) (Figure 2).

For safety reasons, different standard and custom safety protocols have been implemented. The fail-safe use of the lifting axis is guaranteed by a locking cylinder and usage of safety switches. Lifting the robotic arm is only possible at a specific position of the linear axis and at a certain robot pose. Light fences are installed along the housing of the CT-gantry to prevent collisions between the robot and the CT (safety class 2) and are linked into the emergency stop circuit of the system. A light fence and a safety switch (both safety class 4), installed at the entrance doors, complete the standard button-operated emergency stops and ensure intermediate interruption of any robot movement in case of an activated emergency stop. A key-operated bypass of the light fence is also available, which restricts robot motion to allow safe access to the room in order to perform manual operations. The actual system state is displayed via a signal lamp next to the door of the control station room. The maximum robot movement speed has been reduced to an appropriate level as required by the 
application while taking into account the specific safety situation. Movements considered to be safe are performed at higher speeds, than movements during more critical process steps or in more critical areas. A dedicated motion-planning algorithm provides coordinated motion of the robot and external axis. This reduces the motion time of the robot by optimizing the planned robot path, and the algorithm automatically decided whether the robot and the linear axis could move simultaneously or must move consecutively to meet the narrow spatial situation.

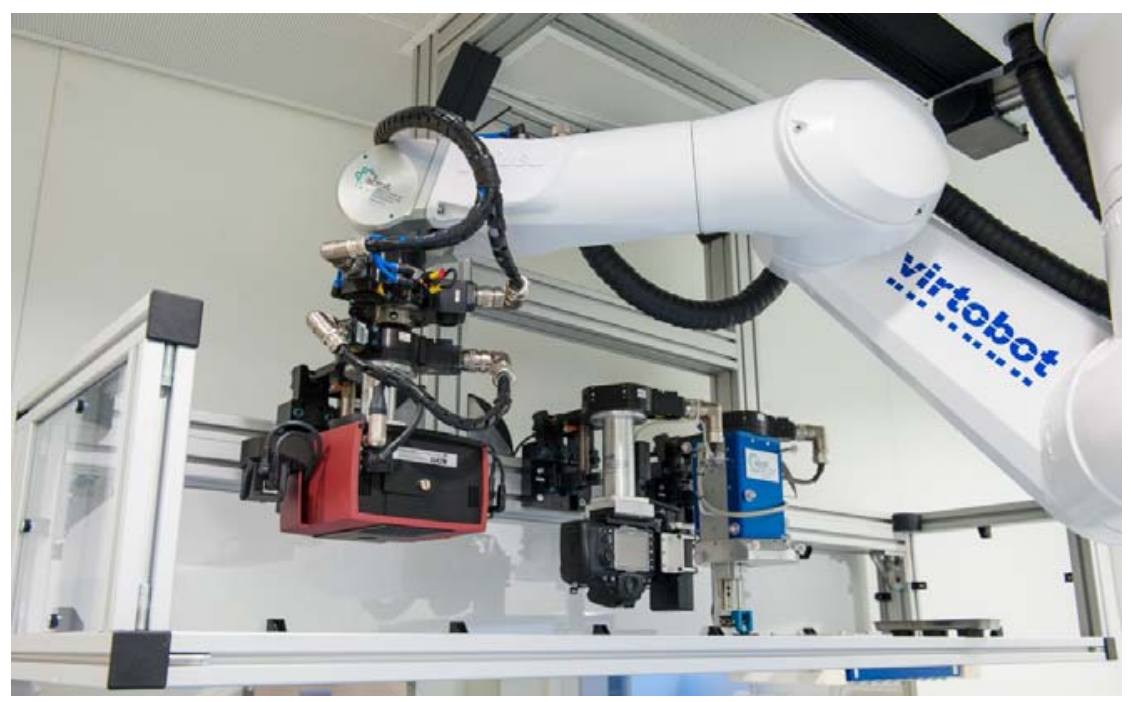

Fig. 2. Tool stand during automatic tool change. Left to right: Surface scanner, digital photo camera for photogrammetry and biopsy module.

\subsection{Software}

The entire software packages for controlling and monitoring the functions of the Virtobot system, run on one control station computer under Windows 7. The Virtopsy-Control-Center (VCC) software coordinates all other software packages, which are required within the Virtopsy workflow, and acts also as a robot master controller. All dedicated programs are remotely controlled and managed by using network interfaces. The performed Virtopsy workflow can be logged and all captured data is stored. The application software ATOS Professional V7.5 SR2 (GOM mbH, Braunschweig, Germany) operates the photogrammetry camera and the $3 \mathrm{D}$ surface scanner, and processes the collected data. It also calculates the fully textured true to scale $3 \mathrm{D}$ polygon model of the surface of the skin. The software can also be used to calibrate the scanner if the lenses have been changed or the accuracy of the system is decreasing. For a later reconstruction it is an essential inspection tool.

\subsection{Photogrammetry and 3D surface scanning}

3D surface documentation is required in cases, where patterned injuries need to be documented. Corpses that are burned or are in a higher stage of decomposition are usually excluded.

Before starting with the 3D documentation of external injuries the deceased must be prepared properly. Depending on the individual case, the preparatory work includes the following tasks. In order to reduce scanning artifacts, the body has to be cleaned of blood, water and other liquids. Additionally, hair has to be removed at the areas of interest. Emergency medical devices on the body should be removed and wounds should be closed to document the original wound pattern, according to needs and objectives. The corpse is placed on the CT table with feet pointing towards the CT-gantry. The body is fixed by using a vacuum mattress to avoid movement during the documentation procedure and allow for later fusion of the surface data with data from the subsequent CT scan (Figure 3).

Circular reference targets are applied randomly onto the skin surface and around the injuries. Two boards with integrated ring coded marker, which supports the photogrammetric calculation, and two scale bars are attached to the side rails of the CT table. By using digital close-range photogrammetry 3D coordinates of the reference targets are being calculated [14]. For that purpose the Virtobot automatically takes photographs from twenty-five predefined perspectives with a calibrated digital single-lens reflex (DSLR) camera Nikon D700 (Nikon Corporation, Tokyo, Japan) using a wide-angle lens AF Nikkor $20 \mathrm{~mm} f / 2.8 \mathrm{D}$ (Nikon Corporation, Tokyo, Japan). The camera positions for calibration and photogrammetric photographs are selected in the VCC software, which guides the operator through the Virtopsy workflow. All taken images are sent to the control station computer using a 
wireless Local Area Network (LAN) interface (Nikon WT-4, Nikon Corporation, Tokyo, Japan), and are automatically forwarded to the ATOS software. The images are processed in the photogrammetric subprogram and $3 \mathrm{D}$ coordinates are calculated automatically from the reference targets, knowing the projection equations of the used optical elements. Later these data are used to automatically fuse the single surface scans. Additionally, the photographs are also utilized as textures for the polygon mesh, which is generated as a result of the surface scan procedure.

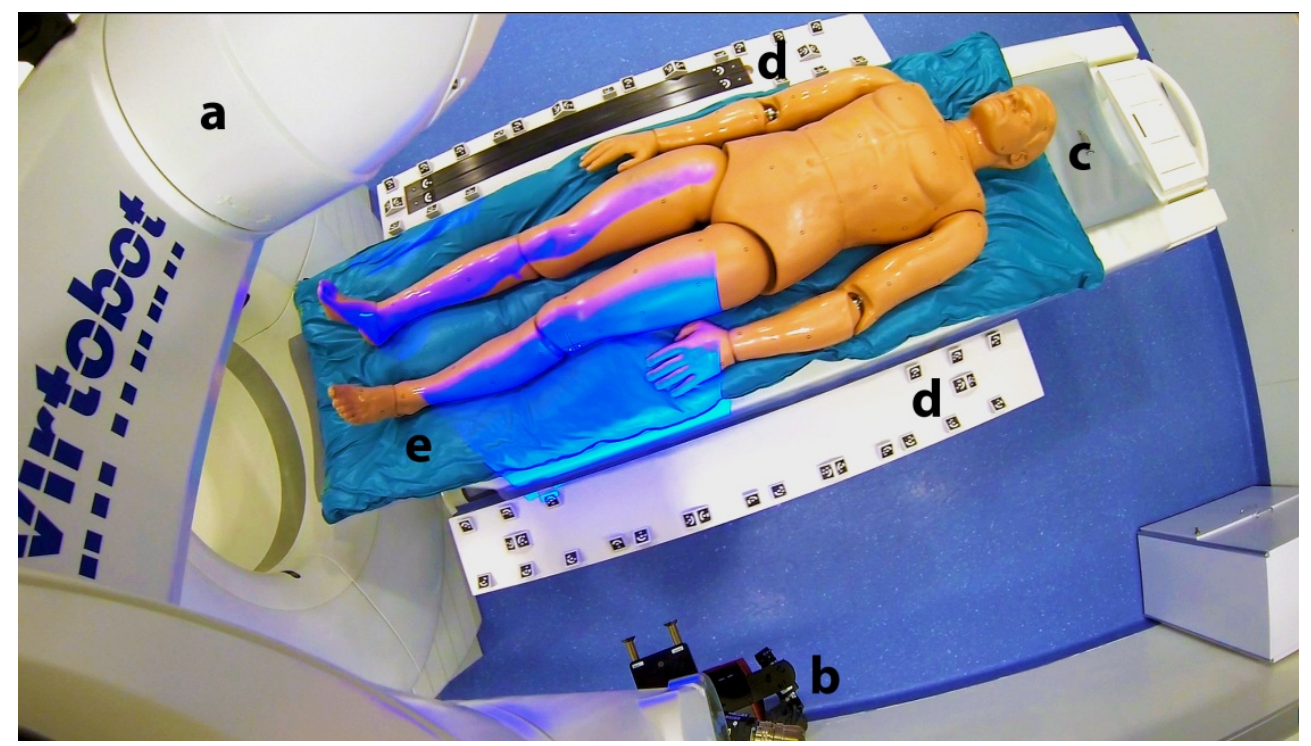

Fig. 3. Setting for surface documentation. a: Virtobot; b: mounted optical 3D surface scanner; c: CT-table;

$d$ : attached side boards with integrated markers and scale bars; e:deceased placed on a vacuum mattress.

In a second step the Virtobot is equipped with an optical surface scanner (Atos Compact Scan 5M, $\mathrm{GOM} \mathrm{mbH}$, Braunschweig, Germany). This system reproduces the geometry of an object in 3D, true to scale and at a high-resolution. It can be utilized wherever small structures must be resolved with high accuracy. The operator selects predefined positions for automatic scanning in the VCC software and starts the automatic measuring process (Figure 4). A projection unit in the middle of the scanner projects a blue light fringe pattern onto the surface. Two charge-coupled device (CCD) cameras, mounted next to the projector, capture the fringe pattern, which is shifted over the object. The data are transferred directly to the control station computer and the ATOS software calculates up to 5 million object points per single measurement within seconds. Due to the complexity of the body shape and the limited surface scanner measuring volume of $600 \times 450 \times 450 \mathrm{~mm}(\mathrm{LxWxH})$ at least twenty-five single measurements are taken from different positions and are transformed fully automatically into a global coordinate system by using positional information of the reference targets gathered during the photogrammetry phase (Figure 4). The operator has the possibility to control the quality of the generated 3D data after the scanning has finished. If there is a lack of data, resulting in gaps in the surface model, it is possible to manually control the robotic arm via VCC software to provide additional grade of flexibility in choosing special scan or photo positions. In this case the operator has to choose one of the preprogrammed scanning positions, which is close to the desired scanning position as a starting point. After switching to the manual robot control mode in the VCC software a picture of the actual robot pose is displayed, offering an intuitive overview about possible robot movements (Figure 5). The operator selects the desired movement directions to move the robot to the desired scanning position while checking the live image of the scanner. As result of the documentation process, the ATOS software generates a polygon mesh of one side of the body. The skin surface with all external injuries is represented by high-resolution, fully textured polygon data. Directly after the surface documentation, a CT will be performed to document the internal injuries. These 3D data are fused into a whole body model of the deceased (Figure 6). In combination with 3D data of the injury inflicting instruments and incident scene, this allows for reconstructions of the course of events of crimes or accidents (Figure 7, Figure 8). 


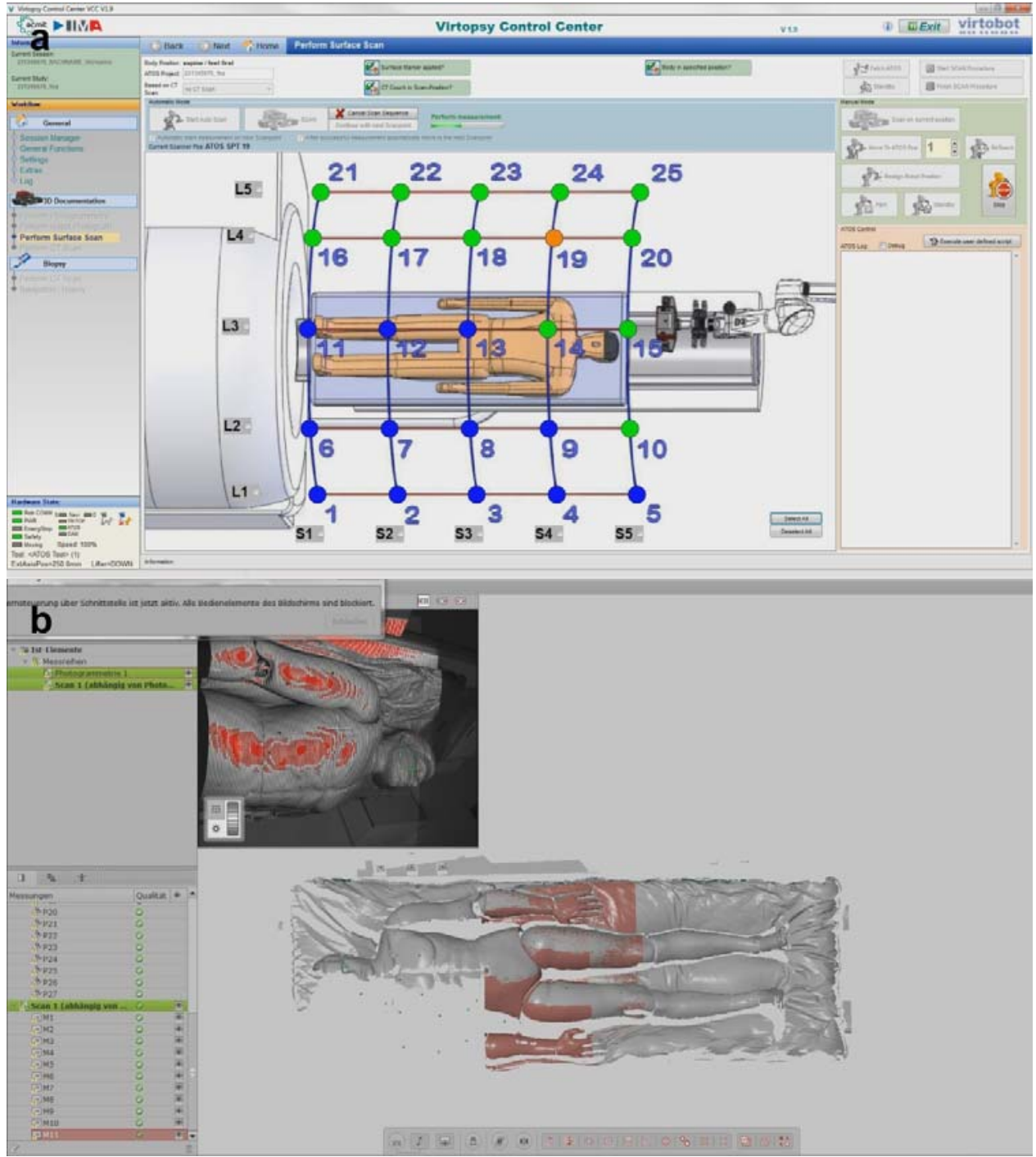

Fig. 4 Screenshots of software packages in use for surface documentation. a: Virtopsy-Control-Centre software (VCC): selection of scanning positions for surface scan. b: Screenshot ATOS Professional Software: 3D model composed of several single surface scans.

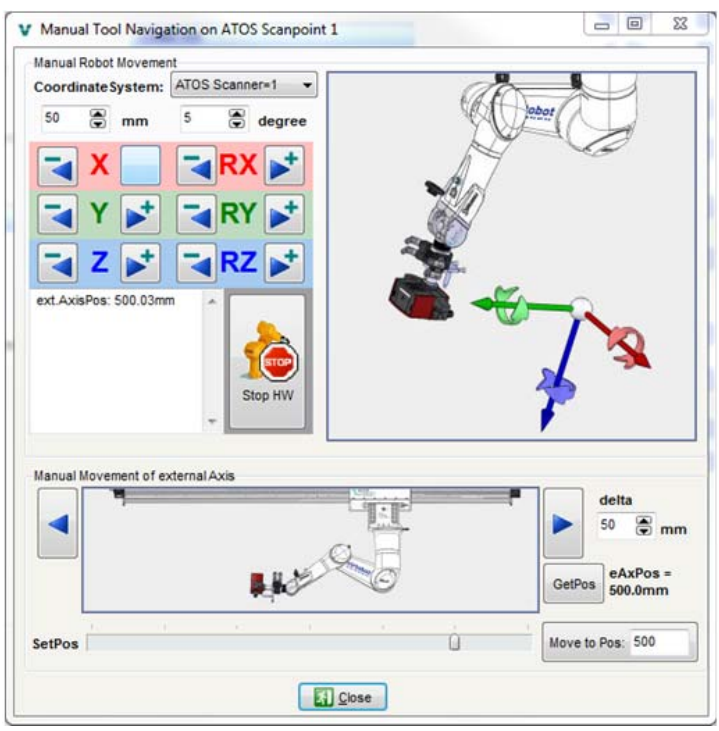

Fig. 5 VCC software dialog for manual positioning of the robotic arm. 

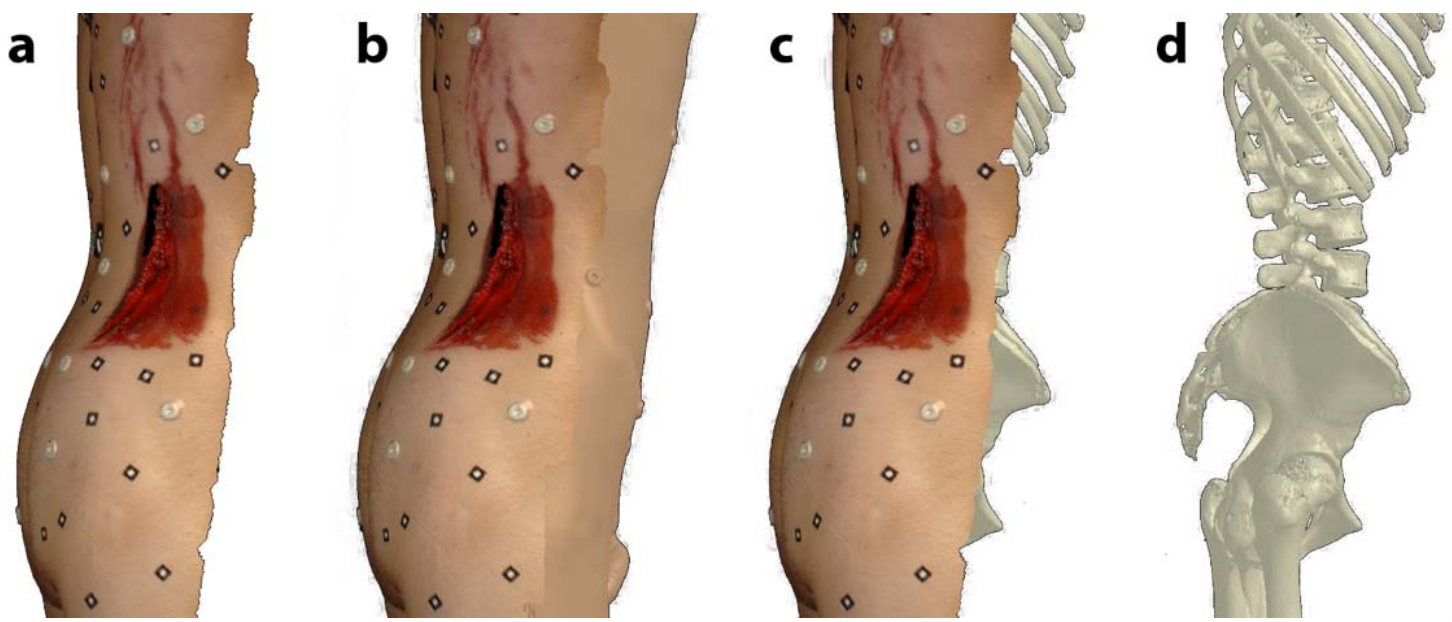

Fig. 6 The 3D model of the external injury on the back and the osseous structure are fused to a real-data-based all-in-one 3D model of the abdomen region. a: Skin model of the injury on the back in color, generated from photogrammetry and surface scanning. b: Skin model of the back fused with the CT skin model of the front side. $c$ : Skin model of the back fused with the CT bone model. $d$ : Bone model generated from the CT images.
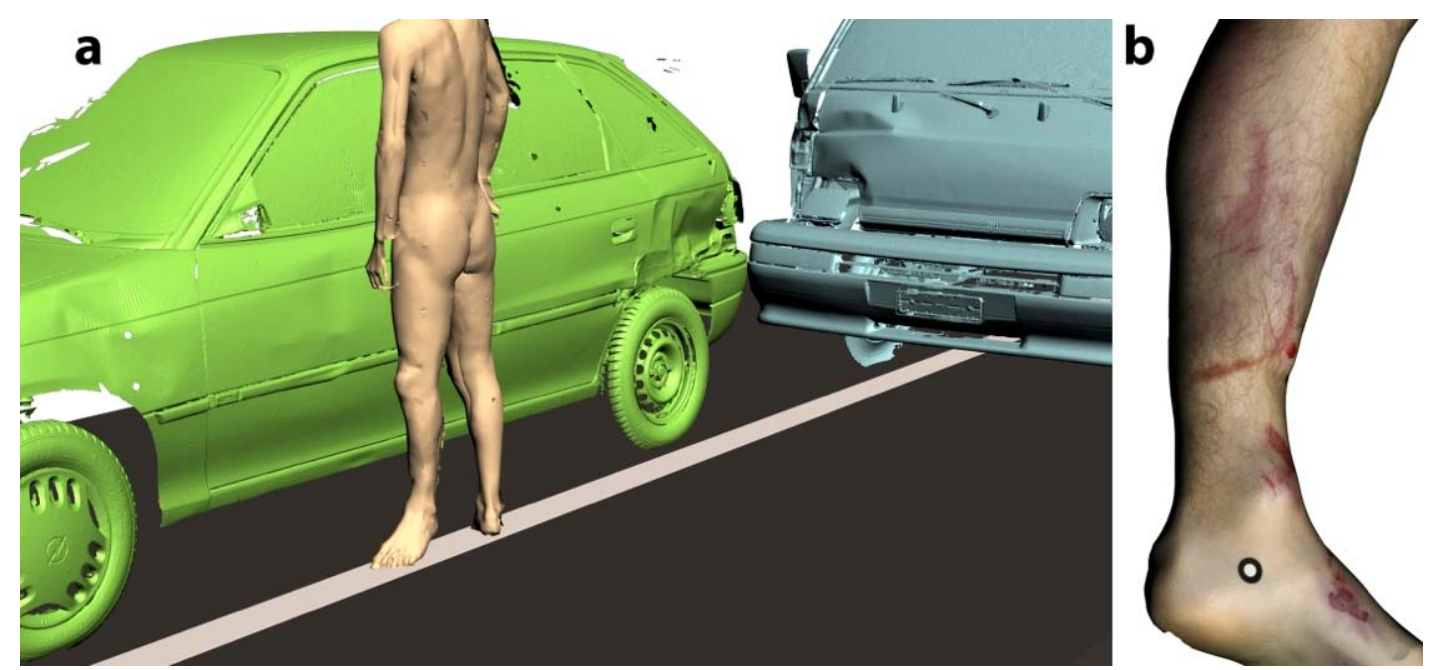

Fig. 7 Traffic accident reconstruction. A person was crushed between his own car and another one. a: Due to the traces on the car and the injuries on the left lower leg, the position of the deceased person to his own car could be reconstructed. b: Real-data-based 3D model of the left lower leg.

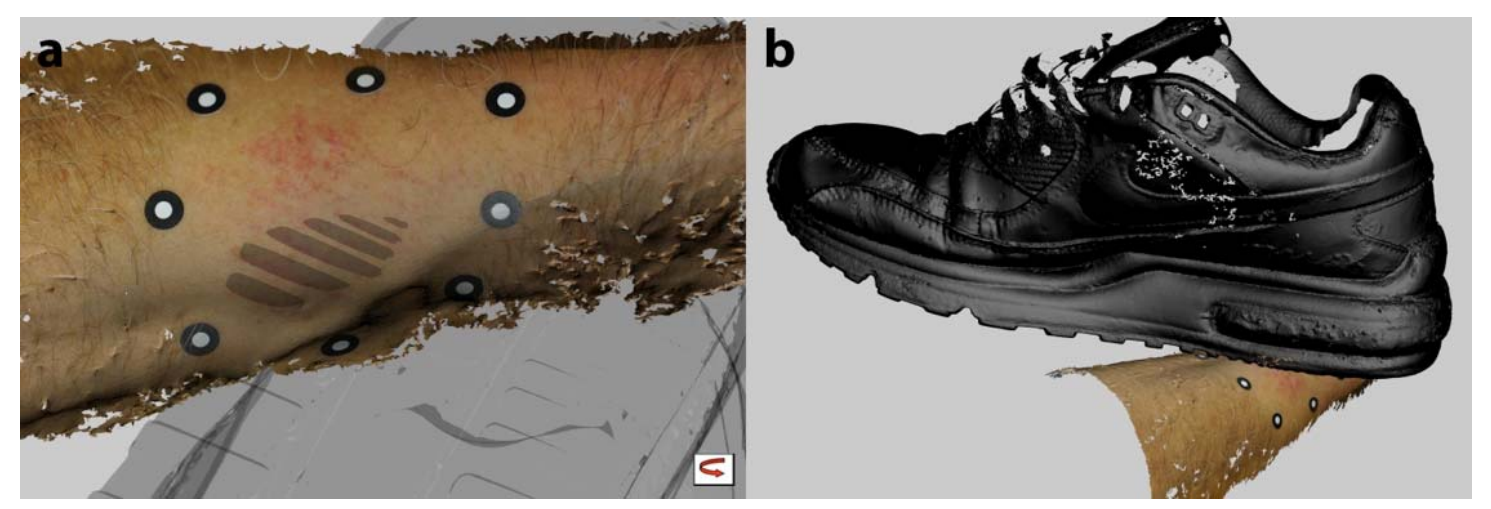

Fig. 8 3D match analysis of the patterned injury on the forearm and the suspected injury-causing sole of a shoe. a: The shape of the patterned injury matched with the shape of the sole. $b$ : The events from perspective. 


\section{Results}

The Virtobot system in Zurich is used in daily routine and has provided robotic assistance for the automated surface documentation of 24 cases over the past 11 months. The prosecutor in charge of these cases explicitly requested the surface documentations. These cases included 17 victims with blunt force injuries, 6 gunshot wounds and 1 case of sharp trauma. In 7 of these cases, a 3D scene reconstruction was requested by the prosecutor, and in the other cases, the data were used for detailed documentation purposes. Concurrently, 475 cases have been submitted to the Institute for post-mortem examinations with CT scans and autopsy reports; therefore, up to now $5.1 \%$ of our cases required surface documentation. By using the robotic assistance, the required photographs and surface scans from a variety of perspectives were made automatically. Therefore only one experienced operator is necessary for the documentation process. All in all several workflow optimizations decrease the time of photogrammetry and 3D surface scanning of one side of a corpse from approximately 40 to 11 minutes. This means a saving of more than $70 \%$ for the mere documentation of the external injuries.

\section{Discussion}

In this article, we present the latest version of the Virtobot system. This model supports fast and accurate, photogrammetric and 3D measurement capabilities, an automatic tool-change mechanism, a new software setup, and different workflow optimizations.

The Virtobot is routinely used for surface documentation at our institute. Approximately $5 \%$ of the deceased fulfill the requirements for whole body surface and wound documentation, and $1.5 \%$ serves as raw material for virtual $3 \mathrm{D}$ reconstructions. While the Virtobot reduces overall scanning times, the entire documentation procedure is still time consuming. This includes the preparation of the corpse (i.e., cleaning, shaving, positioning the body on the vacuum mattress, and application of reference targets), documentation of the entire surface, manual, high-resolution digital photos of wounds and two CT scans (i.e., supine and prone position). By adding all the steps together it takes approximately 2 to 3 hours for the whole body documentation, depending on the individual case. However, because the caseload is approximately 30 surface documentations a year, these times are feasible for our daily routine work. With higher case numbers, there is substantial potential to reduce the overall time. Improving the preparation process can make some of the greatest savings. Instead of manually attaching reference targets onto the body, the known position of the robot during surface scans could be used to merge the single surface scans together, to eliminate this task.

Due to the extensive automation the handling of the Virtobot and the optical surface scanning in combination with photogrammetry is very user-friendly and can be easily operated from one person after a short introduction and training.

The VCC software mainly relies on preprogrammed scanning positions. If additional perspectives are required, i.e., because of missing 3D data, it is possible to manually control the robotic arm via VCC software. For easier handling, it would be desirable to be able to directly define new scanning positions inside the ATOS software.

\section{Conclusion}

Using the Virtobot in combination with the photogrammetry camera and the optical 3D surface scanner is a potentially valuable tool for case documentation in forensic investigations. In conjunction with modern cross-sectional imaging techniques such as CT and MRI, internal and external 3D data can be fused into a whole body model. Coupled with 3D data of the injury-causing instrument and incident scene, it is possible to perform a comprehensive 3D reconstruction that can give important clues to the course of events of crimes or accidents. With the Virtobot system we improved the efficiency of our workflow and increased the level of automation. The robotic system is sufficiently versatile to be adapted to different additional tasks in the future, apart from surface documentation and image guided needle placement.

\section{Conflict of Interest}

Integrated Microsystems Austria $\mathrm{GmbH}$ is planning to commercialize the Virtobot system. 


\section{References}

1. Baglivo M., Winklhofer S., Hatch GM., Ampanozi G., Thali MJ., Ruder TD., (2013): "The rise of forensic and post-mortem radiology-Analysis of the literature between the year 2000 and 2011", Journal of Forensic Radiology and Imaging, Vol.1, No.1, pp. 3-9.

2. Thali MJ., Braun M., Brüschweiler W., Dirnhofer R., (2000): "Matching tire tracks on the head using forensic photogrammetry", Forensic science international, Vol.113, No.1, pp. 281-287.

3. Brüschweiler W., Braun M., Dirnhofer R., Thali MJ., (2003): "Analysis of patterned injuries and injury-causing instruments with forensic 3D/CAD supported photogrammetry (FPHG): an instruction manual for the documentation process", Forensic Science International, Vol.132, No.2, pp. 130-138.

4. Thali MJ., Braun M., Brueschweiler W., Dirnhofer R., (2003): “Morphological imprint': determination of the injury-causing weapon from the wound morphology using forensic 3D/CAD-supported photogrammetry", Forensic Science International, Vol.132, No.3, pp. 177-181.

5. Thali MJ., Braun M., Wirth J., Vock P., Dirnhofer R., (2003): "3D surface and body documentation in forensic medicine: 3-D/CAD Photogrammetry merged with 3D radiological scanning”, Journal of forensic sciences, Vol.48, No.6, pp. 1356-1365.

6. Thali MJ., Braun M., Dirnhofer R., (2003): "Optical 3D surface digitizing in forensic medicine: 3D documentation of skin and bone injuries", Forensic Science International, Vol.137, No.2-3, pp. 203-208.

7. Thali MJ., Braun M., Buck U., Aghayev E., Jackowski C., Vock P., Sonnenschein M., Dirnhofer R., (2005): "VIRTOPSY--scientific documentation, reconstruction and animation in forensic: individual and real 3D data based geo-metric approach including optical body/object surface and radiological CT/MRI scanning", Journal of forensic sciences, Vol.50, No.2, pp. 428-442.

8. Dirnhofer R., Jackowski C., Vock P., Potter K., Thali MJ., (2006): "VIRTOPSY: Minimally Invasive, Imaging-guided Virtual Autopsy”, Radiographics, Vol.26, No.5, pp. 1305-1333.

9. Buck U., Naether S., Braun M., Bolliger S., Friederich H., Jackowski C., Aghayev E., Christe A., Vock P., Dirnhofer R., Thali MJ., (2007): "Application of 3D documentation and geometric reconstruction methods in traffic accident analysis: With high resolution surface scanning, radiological MSCT/MRI scanning and real data based animation", Forensic Science International, Vol.170, No.1, pp. 20-28.

10. Thali MJ., Dirnhofer R., Vock P., (2008): "The virtopsy approach: 3D optical and radiological scanning and reconstruction in forensic medicine", CRC Press, pp. 159-166, 411-432.

11. Reich C., Ritter R., Thesing J., (2000): "3-D shape measurement of complex objects by combining photogrammetry and fringe projection”, Optical Engineering, Vol.39, No.1, pp. 224-231.

12. Subke J., Wehner H-D., Wehner F., Szczepaniak S., (2000): "Streifenlichttopometrie (SLT): a new method for the three-dimensional photorealistic forensic documentation in colour", Forensic science international, Vol.113, No.1, pp. 289-295.

13. Ebert LC., Ptacek W., Naether S., Fürst M., Ross S., Buck U., Weber S., Thali M., (2010): "Virtobot--a multi-functional robotic system for 3D surface scanning and automatic post mortem biopsy", The International Journal of Medical Robotics + Computer Assisted Surgery: MRCAS, Vol.6, No.1, pp. 18-27.

14. 13. Luhmann T., Robson S., Kyle S., Harley I., (2006): "Close range photogrammetry: principles, techniques and applications", Whittles Publishing, Dunbeath. 